\title{
Evolution of Goss Orientation during Thermal Heating with Different Heating Rate for Primary Recrystallization in Grain- oriented Electrical Steel
}

\author{
No-Jin PARK, ${ }^{1) *}$ Hyung-Don $\mathrm{JOO}^{21}$ and Jong-Tae PARK ${ }^{21}$ \\ 1) School of Advanced Materials \& System Engineering, Kumoh National Institute of Technology, Gumi, Rep. Korea. \\ 2) Electrical Steel Research Group, Technical Research Laboratories, POSCO, Pohang, Rep. Korea.
}

(Received on June 19, 2012; accepted on September 14, 2012)

\begin{abstract}
In order to understand how exact Goss-oriented grains form in electrical steel, we studied Goss orientation and microstructure during primary recrystallization using electron back-scattered diffraction. In particular, we examined the effect of heating rate $\left(20^{\circ} \mathrm{C} / \mathrm{s}\right.$ and $\left.150^{\circ} \mathrm{C} / \mathrm{s}\right)$ on the primary recrystallization behavior. In a cold rolled sheet, the Goss fraction in the outer layer is more than twice that in the inside layer, particularly because the fraction of Goss $\pm 5^{\circ}$ grains is much greater at the surface compared with the inner part. When primary recrystallization is completed, the fraction of grains that have Goss $\pm 5^{\circ}$ and $\pm 10^{\circ}$ orientations in the outer and inner layer appear to be similar regardless of the heating rate, and the fraction of Goss $\pm 15^{\circ}$ grains appears to be higher when the sheet is subjected to a rapid heating rate than when it is subjected to a typical heating rate. In addition, the number of Goss $\pm 5^{\circ}$ grains corresponding to a rapid heating rate is twice that corresponding to a typical heating rate, with smaller grain sizes and a more uniform distribution.
\end{abstract}

KEY WORDS: grain-oriented electrical steel; Goss orientation; primary recrystallization; rapid heating process; OIM (orientation image mapping).

\section{Introduction}

Because the anisotropy of magnetic properties in grainoriented electrical steel is very important, there have been numerous studies on the topic. There has been continuous investigation on the control of the microstructure and texture of the electrical steel by adding alloying elements and subjecting it to manufacturing and heat treatment as well as on increasing the magnetic flux density and permeability of the steel while lowering the core loss. ${ }^{1-5)}$ In order to improve on these investigations from the perspective of the texture, it is essential for the Goss orientation $(\{110\}<001>$, that is, the rolling direction is parallel to the $<100>$ direction and the rolling plane is parallel to the $\{110\}$ plane) to have a very high density. It has been reported that heating rate influences on texture, microstructure and magnetic properties in non-oriented electrical steel. ${ }^{6,7)}$ In addition, several patents have shown that rapid heating rate can improve the magnetic properties in grain-oriented electrical steel. ${ }^{89}$ ) They reported that the rapid heating rate can change texture of primary recrystallized samples and improve the magnetic properties. Therefore, the heating rate is one of the most important factors to control grain structure and texture.

In a previous study ${ }^{10)}$ conducted by the authors of this paper on the changes in development behavior and micro-

* Corresponding author: E-mail: njpark@kumoh.ac.kr DOI: http://dx.doi.org/10.2355/isijinternational.53.125 structural changes in Goss orientation during the process from hot-band annealing to primary recrystallization, it was found that the Goss-oriented grains that are present at about $0.3 \%$ fraction in cold rolled sheet are greater in number in the surface layer than in the middle layer. In addition, with regard to Goss-oriented grains, both the grains that were already in existence before cold rolling and the grains that were formed during cold rolling were identified. Furthermore, when the heating rate was 20 or $150^{\circ} \mathrm{C} / \mathrm{s}$ for the primary recrystallization, the fraction and distribution of Gossoriented grains appeared to differ depending on the heating rate. Specifically, as the heating rate increased, the fraction of Goss grains increased and appeared to be almost the same both near the surface and in the middle zone. These results were obtained using electron back-scattered diffraction (EBSD) with measurements taken over relatively small areas of $9 \mu \mathrm{m} \times 26 \mu \mathrm{m}$. Therefore, some limitations came into play in verifying their validity. As such, in this study, the behaviors of Goss-oriented grains in each of the layers were compared by measuring larger areas of $36 \mu \mathrm{m} \times 150$ $\mu \mathrm{m}$.

Furthermore, in previous studies, Goss-oriented grains with up to $15^{\circ}$ deviation from the exact Goss orientation were accepted as Goss grains; however, the magnetic properties of grain-oriented electrical steel deteriorate as the deviation from the Goss orientation increases. In general, depending on whether the rolling direction is deviated to less than $3^{\circ}$ or $7^{\circ}$ from the $<100>$ direction, the product is 
classified as either a high quality or a conventional grainoriented electrical steel, respectively. ${ }^{11-13)}$ Therefore, it is important to study the behavior of grains with orientations in proximity to the exact Goss orientation, and thus, in this study, we conducted investigations by identifying Goss grains with up to $5^{\circ}, 10^{\circ}$, and $15^{\circ}$ deviation from the exact Goss orientation.

\section{Experimental Procedure}

The material used in this investigation was a vacuummelted $\mathrm{Fe}-3.1 \% \mathrm{Si}$ electrical steel (C: $0.06 \%$, Mn: 0.1\%, P: $0.02 \%)$. The steel was initially in the form of an annealed hot-band with a thickness of $2.0 \mathrm{~mm}$ and was subsequently cold rolled to $0.3 \mathrm{~mm}$ in thickness. The primary crystallization was carried out both at the typically applied heating rate of $20^{\circ} \mathrm{C} / \mathrm{s}$ and at a rapid rate of $150^{\circ} \mathrm{C} / \mathrm{s}$. In order to heat cold rolled strip, induction heater with different heating rate was used as method of heating for both typically applied and rapid. In order to change heating rate, the input power was changed. Heat was applied up to about $700^{\circ} \mathrm{C}$ and, in order to observe the recrystallization behavior relative to the heating rate, specimens were extracted at different holding times and estimated fractions of recrystallization and investigated at the sample from early stage of recrystallization and also after full recrystallization. The microstructures and X-ray textures resulting from cold rolling and recrystallization processes had already been identified in a previous study, ${ }^{10}{ }^{2}$ and in this study, orientation image mapping (OIM) based on EBSD was used for the analysis.

\section{EBSD Analysis:}

To observe the microstructure, and the fraction and distribution of Goss grains during the recrystallization process, OIM was carried out using an FE-SEM (Hitachi S-4300SE). The OIM was performed on the cross section of the sheet so that the structure from the surface to the middle of the thickness layer could be observed. The results were analyzed using TSL software. For the measurements, an area of $500 \mu \mathrm{m} \times 250 \mu \mathrm{m}$ and a step size of $500 \mathrm{~nm}$ were used for the completed recrystallization specimens and an area of 36 $\mu \mathrm{m} \times 150 \mu \mathrm{m}$ and a step size of $50 \mathrm{~nm}$ were used for the cold rolled and early recrystallization stage specimens. The crystal orientations were expressed using different colors.

\section{Results and Discussion}

Figure 1 shows a part of the OIM for which measurements were taken from a cold rolled sheet for an area of 36 $\mu \mathrm{m} \times 150 \mu \mathrm{m}$ using a $50 \mathrm{~nm}$ step size for each layer. The layers shown are 0-30 $\mu \mathrm{m}$ (surface layer), 30-60 $\mu \mathrm{m}, 60-$ $90 \mu \mathrm{m}, 90-120 \mu \mathrm{m}$, and $120-150 \mu \mathrm{m}$ (middle layer), where red indicates $\{110\}<001>$ Goss orientation (Euler angle: $\left.\left\{90,90,45^{\circ}\right\}\right)$ and blue indicates $\{100\}<001>$ Cube orientation $\left\{0,0,0^{\circ}\right\}$. Also shown are orientations of $\left\{45,0,0^{\circ}\right\}$, $\left\{0,15,45^{\circ}\right\},\left\{0,30,45^{\circ}\right\},\left\{0,45,45^{\circ}\right\},\left\{0,60,45^{\circ}\right\},\left\{0,75,45^{\circ}\right\}$, and $\left\{0,90,45^{\circ}\right\}$ that correspond to an $\alpha$-fiber texture and the orientations of $\left\{60,54.7,45^{\circ}\right\},\left\{75,54.7,45^{\circ}\right\},\left\{90,54.7,45^{\circ}\right\}$ that correspond to a $\gamma$-fiber texture. The tolerance angle was $15^{\circ}$ for each given orientation. It can be ascertained in all layers that there are wide grains with widths of $1-10 \mu \mathrm{m}$ and narrow grains that are elongated in the rolling direction with widths less than $1 \mu \mathrm{m}$. The wide grains mostly have $\alpha$-fiber texture. The narrow and elongated grains mostly have $\gamma$ fiber texture; a micro band is observed parallel to the rolling direction and a shear band is inclined at $20-40^{\circ}$ from the rolling direction.

Goss orientation mostly appears in small grain forms in strongly deformed shear bands. Shown in Fig. 2 are the area fractions of Goss-oriented grains in the outside (surface-75 $\mu \mathrm{m})$ and inside $(75-150 \mu \mathrm{m})$ layers of the cold rolled sheet. Goss grains within tolerance angles of $5^{\circ}, 10^{\circ}$, and $15^{\circ}$ of the exact Goss orientation were denoted as Goss $\pm 5^{\circ}$, Goss $\pm 10^{\circ}$, and Goss $\pm 15^{\circ}$, respectively. On average, the fraction of Goss $\pm 5^{\circ}$ Goss $\pm 10^{\circ}$, and Goss $\pm 15^{\circ}$ grains was $0.017 \%, 0.148 \%$, and $0.438 \%$, respectively, and it was possible to see that the area fraction of Goss $\pm 5^{\circ}$ grain was very small compared to that of Goss $\pm 15^{\circ}$ grain. In addition, in all cases, the Goss fractions in the outside layer of the sheet were about twice those in the inside layer. This, as discussed in the previous studies, ${ }^{10}$ is attributable to the fact that many of the Goss grains had already been developed in the annealed hot-band before cold rolling. In particular, from the fact that the area fraction of Goss $\pm 5^{\circ}$ grain was significantly higher in the outside layer as compared with that in the inside layer, it can be concluded that in order to manufacture a good quality grain-oriented electrical steel, it is important to control the manufacturing process so that these surface Goss grains grow in the inside layer as well. The results also show that the sizes of Goss grains are similar in all layers, except that depending on the deviation from the Goss orientation, as the tolerance angle of Goss orientation increases the grain size increases slightly, from $0.18 \mu \mathrm{m}$ for Goss $\pm 5^{\circ}$ grain to $0.21 \mu \mathrm{m}$ for Goss $\pm 10^{\circ}$ grain and $0.24 \mu \mathrm{m}$ for Goss $\pm 15^{\circ}$ grain.

In order to verify whether the Goss grains existing in the cold rolled sheet were formed by cold rolling or whether they were originally existent in the annealed hot-band, the image quality (IQ) values of Goss $\pm 15^{\circ}$ grains were determined for five separate layers from the surface to the middle layer. Goss $\pm 5^{\circ}$ and $10^{\circ}$ grains were not considered, because their numbers were small and not appropriate for statistical treatment. IQ values are a measure for evaluating the quality of EBSD patterns and are lower in value if there are distortions in a crystal lattice. By using IQ values, it is possible to qualitatively determine strain distribution in the microstructure, ${ }^{14)}$ and the low IQ value indicated that the stored strain energy of grains is high, i.e. in regions with a high EBSD image quality indicating low stored strain energy. ${ }^{15}$ In this study, because OIM was measured over wide areas and a number of times, IQ values were expected to vary depending on the measurement conditions. Therefore, the relative IQ values, rather than the absolute IQ values, provided by the TSL program were analyzed from $0 \%$ to $100 \%$ at $20 \%$ intervals. The strain cannot determine quantitatively yet with the IQ value. This should be investigated in future.

The fractions relating to the relative IQ values of Goss grains in each layer are shown in Fig. 3. It is possible to see that the relative IQ value in the middle layer $(90-150 \mu \mathrm{m})$ is much lower than that of the surface layer $(0-60 \mu \mathrm{m})$. This would mean that most of the Goss grains that form in the middle layer are subjected to stronger deformation. In the 


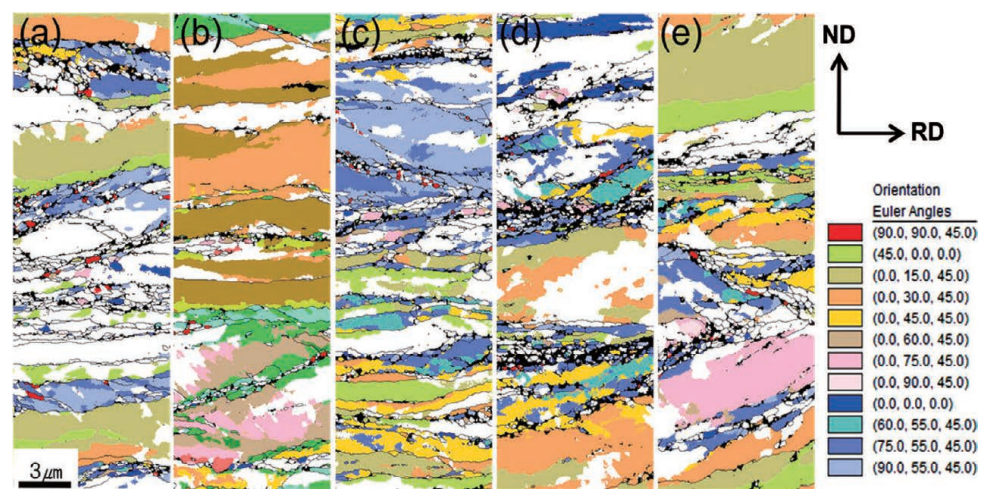

Fig. 1. Orientation mapping of cold-rolled sheet obtained by EBSD with a step size of $50 \mathrm{~nm}$ at (a) $0-30 \mu \mathrm{m}$, (b) $30-60$ $\mu \mathrm{m}$, (c) $60-90 \mu \mathrm{m}$, (d) $90-120 \mu \mathrm{m}$, (e) $120-150 \mu \mathrm{m}$ from the surface, ND-RD section. Colors indicate orientations as in legend.
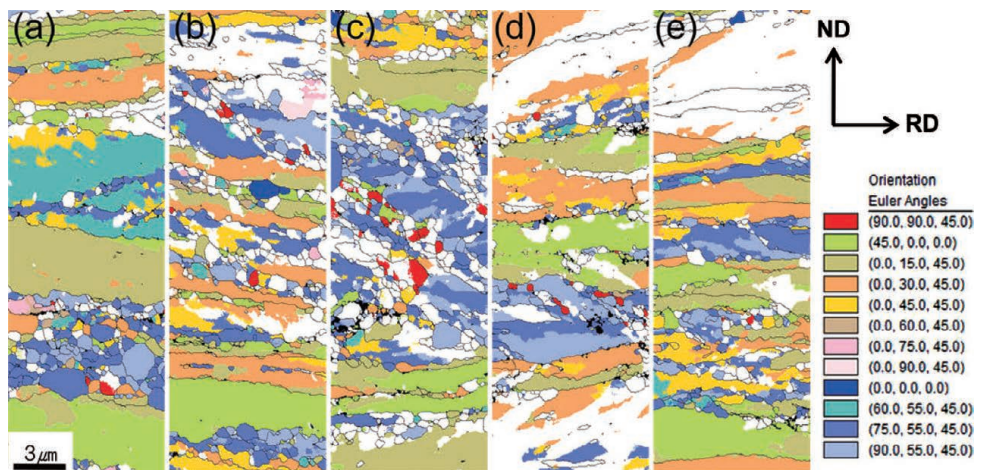

Fig. 5. Orientation mapping of partially recrystallized sheet obtained by EBSD with a step size of $50 \mathrm{~nm}$ at (a) $0-30 \mu \mathrm{m}$, (b) 30-60 $\mu \mathrm{m}$, (c) $60-90 \mu \mathrm{m}$, (d) $90-120 \mu \mathrm{m}$, (e) $120-150 \mu \mathrm{m}$ from the surface, heated with $150^{\circ} \mathrm{C} / \mathrm{s}$, ND-RD section.

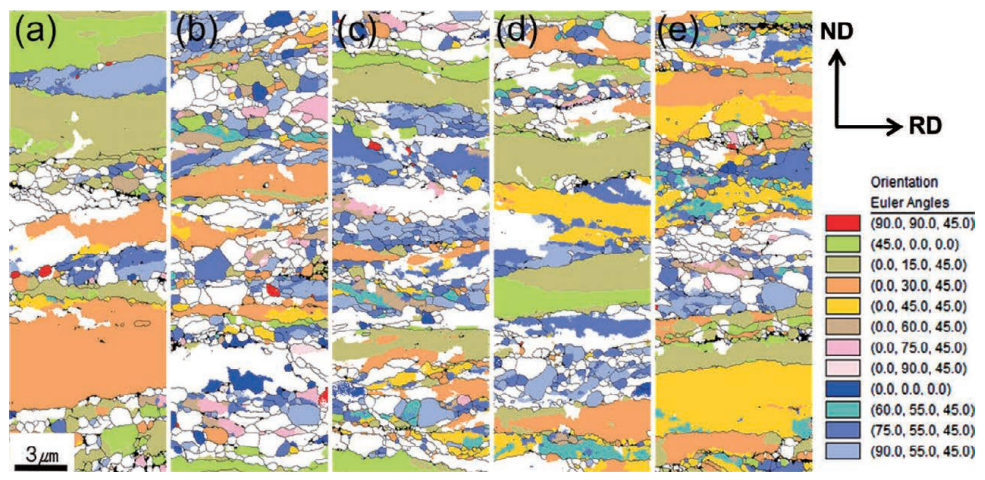

Fig. 6. Orientation mapping of partially recrystallized sheet obtained by EBSD with a step size of $50 \mathrm{~nm}$ at (a) $0-30 \mu \mathrm{m}$, (b) 30-60 $\mu \mathrm{m}$, (c) $60-90 \mu \mathrm{m}$, (d) $90-120 \mu \mathrm{m}$, (e) $120-150 \mu \mathrm{m}$ from the surface, heated with $20^{\circ} \mathrm{C} / \mathrm{s}$, ND-RD section.

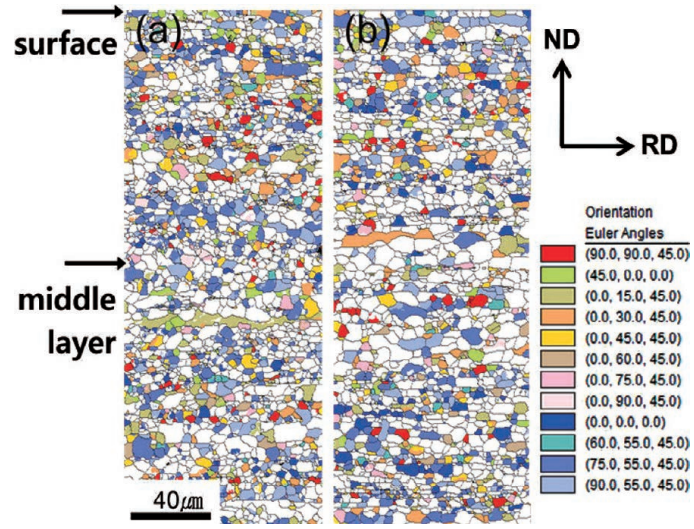

Fig. 10. Orientation mapping of fully recrystallized sheet obtained by EBSD with a step size of $500 \mathrm{~nm}$ through thickness heated at (a) rapid, (b) typical rate, ND-RD section.

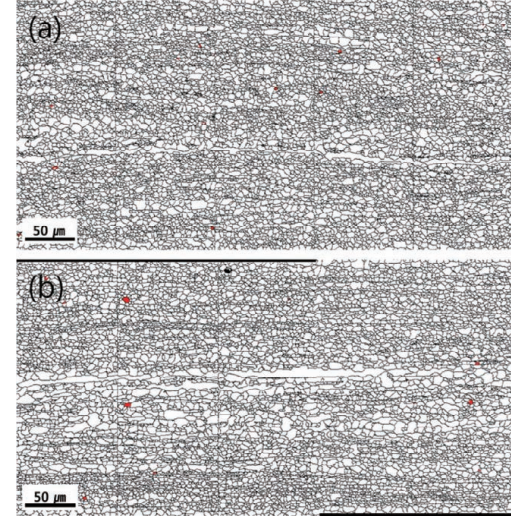

Fig. 12. Distribution of Goss $\pm 5^{\circ}$ grains in total measured area after full recrystallization with (a) rapid and (b) typical heating rate. Red indicates Goss $\pm 5^{\circ}$ grains. 
$30-60 \mu \mathrm{m}$ layer, $11.4 \%$ of the areas have relative IQ values over $60 \%$, which indicates that there were many pre-existing Goss grains that formed during the hot-rolling and hot-band annealing. In order to compare these results with grains that have other orientations, the fraction taken up by the relative IQ value of the total number of grains is shown in Fig. 4 . The relative IQ value of all grains shows a higher value than that of the Goss grains. This result matches the previously published reports that used X-ray and electronic microscopy

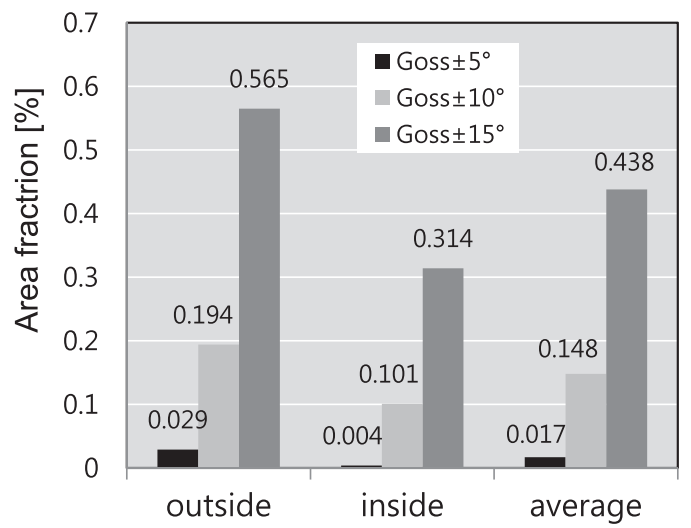

Fig. 2. Area fraction of Goss-oriented grains in the outside (0-75 $\mu \mathrm{m}$ from the surface) and inside (75-150 $\mu \mathrm{m}$ from the surface) layers of the sheet after cold rolling. Goss grains are denoted $\pm 5^{\circ}, \pm 10^{\circ}$, and $\pm 15^{\circ}$ according to their spread angle from the perfect Goss orientation.

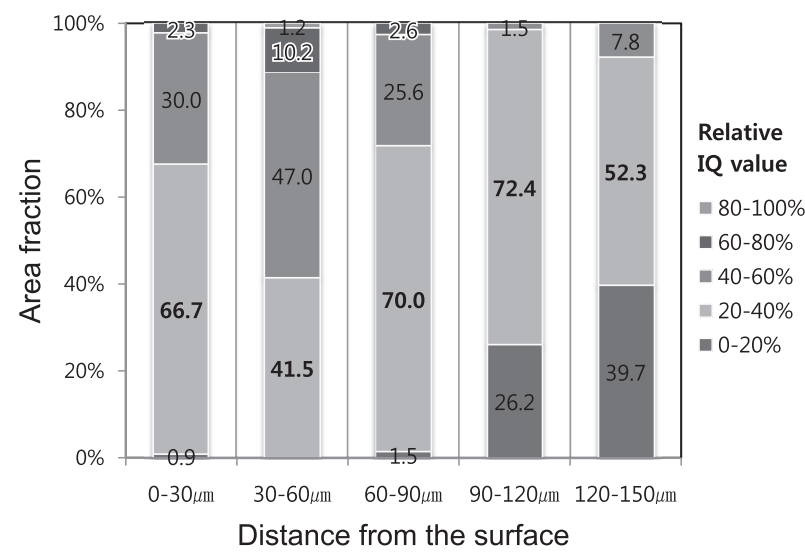

Fig. 3. Fraction of relative IQ value of Goss-oriented grains in the different layers

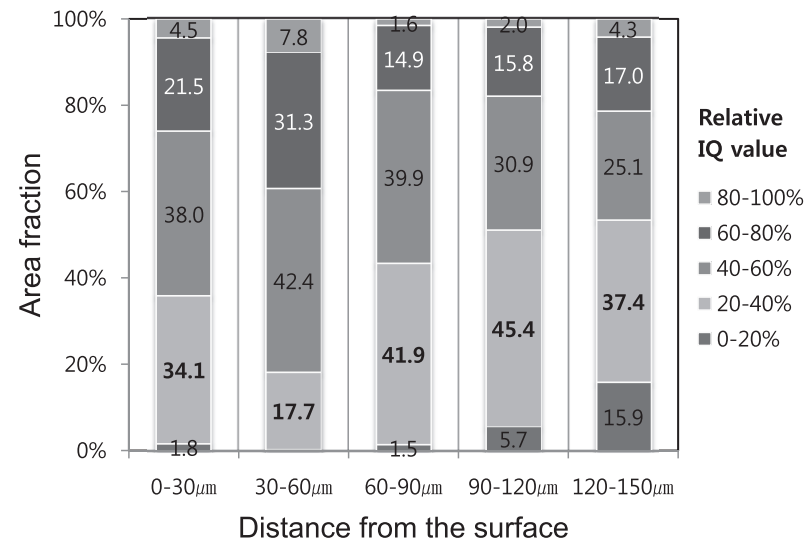

Fig. 4. Fraction of relative IQ value of all grains in the different layers. and stated that in cold rolled steel sheet the Goss grains have much higher stored energy than grains of other orientation. ${ }^{13,16-18)}$ In addition, the relative IQ value of all grains in the middle layer shows lower value than those in the surface layer; however, as in the case of the Goss grains, the difference is not that severe. This result indicates that during cold rolling the Goss grains were more deformed compared with the grains of other orientation; in particular, the Goss grains of the middle layer were subjected to more severe deformations. This result proves that Goss grains are present mostly in shear bands that have severe deformation. In addition, the reason why the IQ values of the surface to $60 \mu \mathrm{m}$ layer are higher than the middle layer is thought to be that at the surface, instead of the grains being compressed due to the plane strain deformation, they rotate due to the shear deformation, thereby causing less lattice strain to be accumulated.

Figures 5 and 6 show parts of the OIM of the partially recrystallized sheet, measured over an area of $36 \mu \mathrm{m} \times 150$ $\mu \mathrm{m}$ with a step size of $50 \mathrm{~nm}$. The observed recrystallization fractions were different for the inside and outside layers; in the case of the rapid heating rate the inside layers reached $21.5 \%$ recrystallization while the outside layers reached $39.9 \%$ recrystallization, while in the case of the typical heating rate the insides reached $25.4 \%$ recrystallization and the outside layers reached $40.0 \%$ recrystallization. The reason why the inside and outside layers showed different fractions of recrystallization is thought to be attributable to the fact that recrystallization progressed from the outside to the inside due to the basic nature of induction heating, which the eddy currents are produced on the outside of the work piece in what is often referred to as "skin effect" heating. ${ }^{19)}$ Due to this skin effect, outside layer of sample heat more quickly than inside of sample and the outside layer of sample might have a little bit higher temperature than inside of that.

In Fig. 7, the area fractions of Goss grains in the outside and inside layers of the sheet after partial recrystallization are shown. Depending on the heating rate, large differences were observed and, in the case of recrystallization via a rapid heating rate, more Goss grains were observed than in cold rolled specimens. In particular, the area fraction of Goss $\pm 5^{\circ}$ grain in the inside of the sheet was high (compared with Fig. 2 ). However, when the heating rate is $20^{\circ} \mathrm{C} / \mathrm{s}$, it can be observed that smaller fractions of Goss grains with are apparent than in the cold rolled specimens, and this is a result of the fact that many of the Goss grains formed in cold

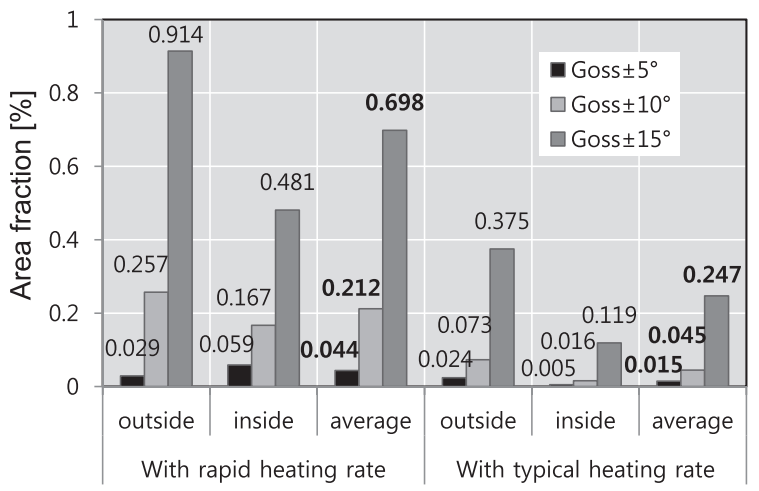

Fig. 7. Area fraction of Goss-oriented grains in the outside (0-75 $\mu \mathrm{m})$ and inside $(75-150 \mu \mathrm{m})$ layers of the sheet after partial recrystallization with rapid and typical heating rate. 
rolling are destroyed during the early stage of recrystallization in the case of typical heating rates.

Shown in Fig. 8 are the area fractions of relative IQ values of Goss-oriented and all grains in the outside and inside layers after partial recrystallization at a rapid heating rate. Unlike the cold rolled specimens, the IQ values of the Goss grains appear higher than the value for all grains. This shows that the Goss grains, which had accumulated more strain during cold rolling, were released faster during recrystallization than grains with other orientations, and this tends to occur more strongly in the outside layer. The area fractions of IQ values of Goss-oriented and all grains in the outside and inside layers after partial recrystallization at a typical heating rate are shown in Fig. 9. Similar to the specimens for which the temperature rise was rapid, the IQ values of the Goss grains are higher than that of grains with other orientation, and the average Goss grain IQ value is higher than in the case where the heating rate is rapid. In addition, the IQ value of Goss grains in the inside appears higher when the heating rate is typical.

Through these results, it can be ascertained that behavior during the early stage of recrystallization is closely associated with the crystal orientation and heating rate. Goss grains initiate faster than grains with other orientation regardless of the heating rate, however, if the heating rate is rapid, recrystallization of the Goss grains on the surface begins more quickly than in cases when the heating rate is slow, and the inner portions progress slowly.

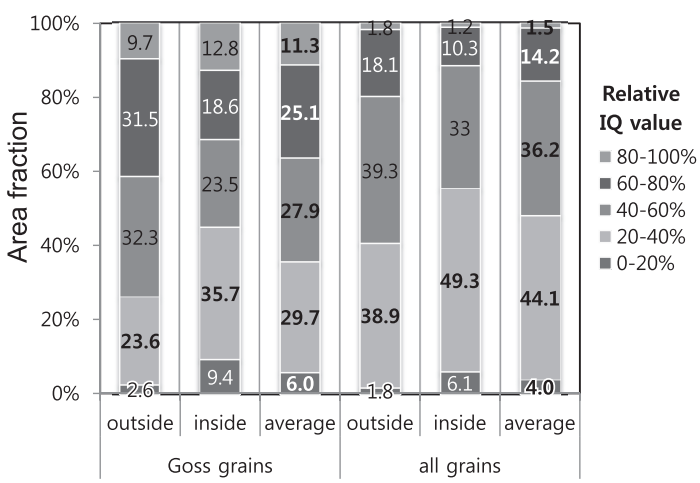

Fig. 8. Area fraction of relative IQ value of Goss-oriented and all grains in the outside $(0-75 \mu \mathrm{m})$ and inside $(75-150 \mu \mathrm{m})$ layers after partial recrystallization at the rapid heating rate.

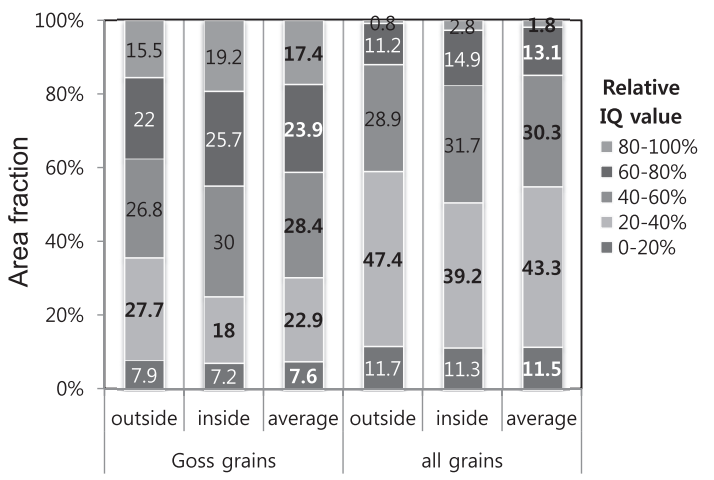

Fig. 9. Area fraction of relative IQ value of Goss-oriented and all grains in the outside $(0-75 \mu \mathrm{m})$ and inside $(75-150 \mu \mathrm{m})$ layers after partial recrystallization at the typical heating rate.
Shown in Fig. 10 is part of the OIM measured from a 500 $\mu \mathrm{m} \times 250 \mu \mathrm{m}$ areas with a step size of $500 \mathrm{~nm}$ when recrystallization is completed with rapid and typical heating rates. It could be seen that in both specimens $100 \%$ recrystallization was achieved in the outside layer and over $98 \%$ recrystallization in the inside layer. In Fig. 11, the area fractions of Goss-oriented grains in the outside and inside layers of the sheet after full recrystallization are shown. The fractions of Goss $\pm 5^{\circ}$ and $\pm 10^{\circ}$ grains do not show any significant differences with respect to the heating rate. However, the increase in the Goss $\pm 15^{\circ}$ grain fraction in the inner part is higher when a rapid heating rate is used than that with a typical heating rate. This is deemed to be attributable to the fact that the heating rate does not increase the area fraction of Goss grain during the primary recrystallization and, in particular, that the heating rate does not contribute to an increase in grains that are in close proximity to the exact Goss orientation. However, when examining the number, distribution, and size of Goss $\pm 5^{\circ}$ grains, it can be seen that there are apparent differences depending on the heating rate. The measured numbers of Goss-oriented grains in the outside and inside layers of the sheet at rapid and typical heating rates are given in Table 1. Even that the number of Goss $\pm 5^{\circ}$ grain seems to be small for the statistical treatment, following statements are explainable qualitatively. In Fig. 12 , Goss $\pm 5^{\circ}$ grains are expressed in red in the microstructures of the specimens for which the temperature was raised at both rapid and typical rates, and in Fig. 13, the heating rate-dependent grain sizes are shown. In the case of the rapid heating rate, 28 grains of an average size of $1.94 \mu \mathrm{m}$ are distributed uniformly throughout the sheet without much difference in their grain size. However, the case of the typical heating rate, 14 grains are present as a mixture of large

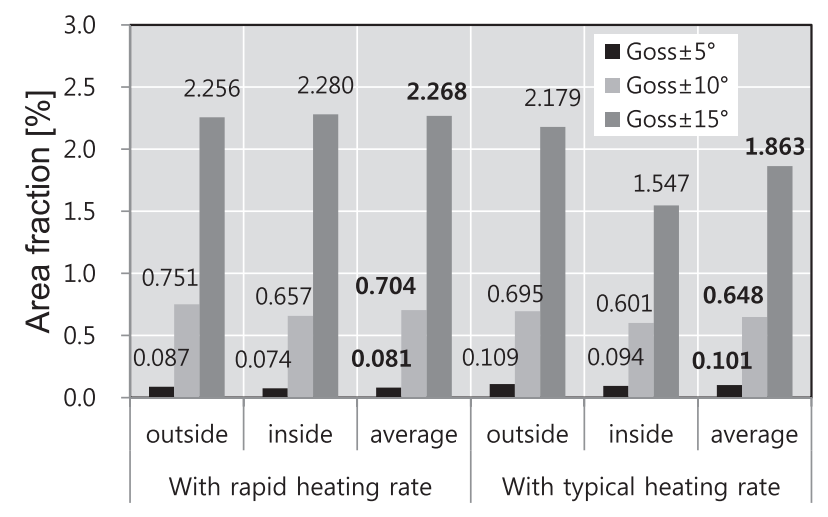

Fig. 11. Area fraction of Goss-oriented grains in the outside (0-75 $\mu \mathrm{m})$ and inside $(75-150 \mu \mathrm{m})$ layers of the sheet after full recrystallization at rapid and typical heating rates.

Table 1. Number of Goss-oriented grains in the outside $(0-75 \mu \mathrm{m})$ and inside $(75-150 \mu \mathrm{m})$ layers of the sheet after full recrystallization at rapid and typical heating rates.

\begin{tabular}{|c|c|c|c|c|}
\hline & \multicolumn{2}{|c|}{ with rapid heating rate } & \multicolumn{2}{|c|}{ with typical heating rate } \\
\hline & outside & inside & outside & inside \\
\hline $\operatorname{Goss} \pm 5^{\circ}$ & 16 & 12 & 9 & 5 \\
\hline $\operatorname{Goss} \pm 10^{\circ}$ & 73 & 73 & 60 & 37 \\
\hline Goss $\pm 15^{\circ}$ & 215 & 224 & 169 & 107 \\
\hline
\end{tabular}




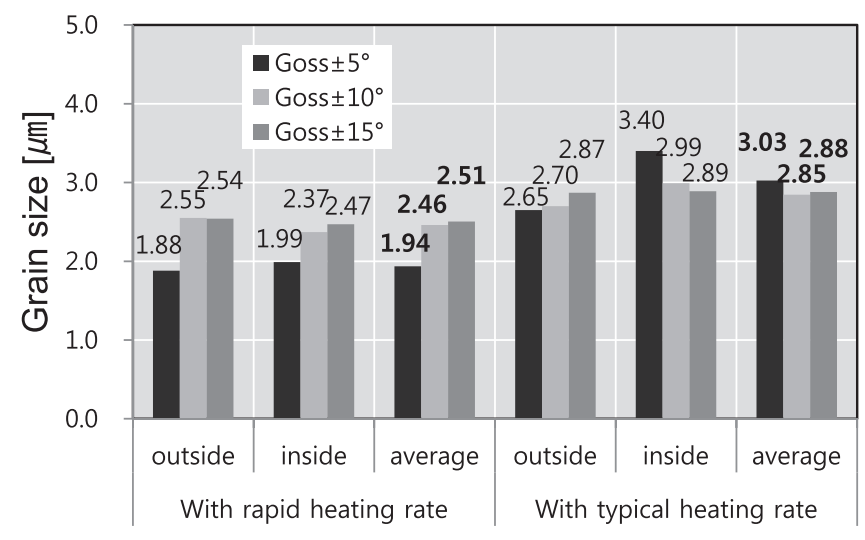

Fig. 13. Grain size of Goss-oriented grains in the outside (0-75 $\mu \mathrm{m})$ and inside $(75-150 \mu \mathrm{m})$ layers of the sheet after full recrystallization at rapid and typical heating rates.

and small grains. With an average grain size of $3.03 \mu \mathrm{m}$, they appear much larger than those in the case of a rapid heating rate. As shown, in cases of a rapid heating rate, although the area fractions of Goss $\pm 5^{\circ}$ grain are similar, there are about twice the number of grains than in cases of a typical heating rate, and it could be seen that the sizes are smaller with a more uniform distribution. As mentioned previously, this is a result of the Goss grains increasing in number without being destroyed during the early stage of recrystallization at a rapid heating rate. As such, it is deemed that the improvement in the magnetic properties that appears during the primary recrystallization stage when the heating rate is rapid is a result of the number, size, and distribution of Goss $\pm 5^{\circ}$ grains exerting influence on the formation of exact Goss grains during the secondary recrystallization.

\section{Conclusions}

By heating cold rolled electrical steel that contained 3.1\% $\mathrm{Si}$ at a typical and a rapid heating rate of 20 and $150^{\circ} \mathrm{C} / \mathrm{s}$, respectively, and by analyzing the recrystallization behavior with EBSD-OIM with respect to the heating rate, the following results were obtained:

(1) In cold rolled specimens, the Goss fractions in the outside layer are more than twice those in the inside layer and, in particular, the fraction of Goss $\pm 5^{\circ}$ grains is much greater at the surface as compared with the inner part. Thus, it is very critical to control and facilitate the growth of surface Goss grains all the way to the inner part in order to manufacture high quality grain-oriented electrical steel.

(2) In cold rolling, Goss grains that are present in the inner layer are subject to more deformation than grains with other orientation. In the surface layer, there are Goss grains that exist prior to cold rolling, which are formed during the hot-rolling and hot-band annealing.

(3) During the primary recrystallization, the fraction of recrystallized Goss grains increases when the heating rate is rapid, and this is more prominent in the outer layer. However, under a typical heating rate, many of the Goss grains are destroyed during the early stage of recrystallization.

(4) When recrystallization is complete, the fraction of Goss $\pm 15^{\circ}$ grains heated at a rapid heating rate appears higher than those in cases of being heated with a typical speed. In addition, the Goss grain sizes after recrystallization are smaller when the heating rate is rapid.

(5) When recrystallization is complete, in cases of a rapid heating rate, the number of Goss $\pm 5^{\circ}$ grains is about twice for a typical heating rate, with smaller sizes and a more uniform distribution.

\section{REFERENCES}

1) Y. Yoshitomi, K. Iwayama, K, Kuroki, Y. Matuo and H. Masui: $J$. Jpn. Inst. Met., 57 (1993), 612.

2) M. Matsuo, T. Sakai, M. Tanino, T. Shindo and S. Hayami: Proc. of 6th Int. Conf. on Textures of Materials, ed. by S. Nagashima, ISIJ, Tokyo, (1981), 918.

3) K. Iwayama and T. Haratani: J. Magn. Magn. Mater., 19 (1980), 15.

4) A. Datta: IEEE Trans. Mag, MAG-12, 6 (1976), 867.

5) Z. Xia, Y. Kang and Q. Wang: J. Magn. Magn. Mater., 320 (2008), 3229 .

6) J. T. Park, J. A. Szpunar and S. Y. Cha: ISIJ Int., 43 (2003), 1611.

7) J. Wang, J. Li, X. Wang, J. Tian, C. Zhang and S. Zhang: J. Iron Steel Res., 17 (2010), 54.

8) US Patent, No. 4898626, (1998).

9) Japanese Patent, No. 1997-256051

10) N. J. Park, E. J. Lee, H. D. Joo and J. T. Park: ISIJ Int., 51 (2011), 975.

11) R. Ricci Bitti and M. Candiotti: J. Magn. Magn. Mater., 26 (1982), 11 .

12) S. Mishra, C. Daermann and K. Luecke: Acta Metall., 32 (1984), 2185.

13) M. Matsuo: ISIJ Int., 29 (1989), 809

14) S. T. Wardle, L. S. Lin, A. Cetel and B. L. Adams: Proc. of 52nd Annual Meeting of the Microscopy Society of America, MSA, New Orleans, (1994), 680

15) D. Dorner, S. Zaefferer and D. Raabe: Acta Mater., 55 (2007), 2519.

16) I. L. Dillamorc, C. J. E. Smith and T. W. Watson: Met. Sci. J., 1 (1967), 49.

17) C. J. E. Smith and I. L. Dillamore: Met. Sci. J., 4 (1970), 161.

18) H. Takechi, H. Katoh and S. Nagashima: Trans. Met. Soc. AIME, 242 (1969), 56.

19) R. E. Haimbaugh: Practical Induction Heat Treating, ASM International, Materials Park, OH, (2001), 5. 